\title{
Robust, reduced-order, nonstrictly proper state estimation via the optimal projection equations with Petersen-Hollot bounds
}

\author{
Wassim M. HADDAD \\ Department of Mechanical Engineering, Florida Institute of Technology, Melbourne, Fl 3290I, USA
}

\author{
Dennis S. BERNSTEIN * \\ Harris Corporation, Government Aerospace Systems Division, MS 22/4848, Melbourne, FL 32902, USA
}

Received 10 March 1987

Revised 11 June 1987

\begin{abstract}
A state-estimation design problem involving parametric plant uncertainties is considered. An error bound suggested by recent work of Petersen and Hollot is utilized for guaranteeing robust estimation. Necessary conditions which generalize the optimal projection equations for reduced-order state estimation are used to characterize the estimator which minimizes the error bound. The design equations thus effectively serve as sufficient conditions for synthesizing robust estimators. An additional feature is the presence of a static estimation gain in conjunction with the dynamic (Kalman) estimator, i.e., a nonstrictly proper estimator.
\end{abstract}

Keywords: Robust Kalman filter, Error bounds, Reduced-order state estimation.

\section{Introduction}

As is well known $[2,5-8,11,14,15]$ optimal filters based upon nominal parameter values may be severely degraded in the presence of parameter deviations. Thus, it is desirable to obtain robust state estimators which provide acceptable performance over the range of parametric uncertainty. The approach of the present paper is related to the guaranteed cost approach developed for control in $[4,16]$ and applied to estimation in [11]. Specifically, the main idea is to bound the effect of the uncertain parameters on the estimation error over the uncertainty range and then choose estimator gains to minimize the estimation bound. Thus the actual estimation error is guaranteed to lie below the prescribed upper bound.

The technique used to determine minimizing estimator gains is based upon a generalization of the optimal projection equations for reduced-order state estimation [1]. Thus the results of the present paper effectively extend the results of [1] to the case of system uncertainties. It should be noted that the optimal projection equations, which are necessary conditions for optimality, now serve as sufficient conditions for robust estimation by virtue of the fact that a bound on the estimation error is being minimized rather than the estimation error itself. The bound utilized in the present paper is an extension of the approach developed in $[12,13]$ for constructing Lyapunov functions for full-state feedback and utilized in [10] to characterize the structured stability radius.

An additional feature of the present paper is the inclusion of a static feedback gain in conjunction with the dynamic estimator. Thus the results of the present paper represent a generalization of standard results to the case of nonstrictly proper estimation.

* Supported in part by the Air Force Office of Scientific Research under contract F49620-86-C-0002. 


\section{Notation and definitions}

Note: All matrices have real entries.

$\mathbb{R}, \mathbb{R}^{r \times s}, \mathbb{R}^{\mathrm{r}}, \mathbb{E} \quad$ real numbers, $r \times s$ real matrices, $\mathbb{R}^{r \times 1}$, expected value.

$I_{r},()^{\mathrm{T}}$

$\mathbb{S}^{r}, \mathbb{N}^{r}, \mathbb{P}^{r}$

$r \times r$ identity matrix, transpose

$Z_{1} \leq Z_{2}, Z_{1}<Z_{2}$

$n, l, \hat{l}, n_{\mathrm{e}}, p, q ; \tilde{n}$

$r \times r$ symmetric, nonnegative-definite, positive-definite matrices.

$x, y, \hat{y}, y_{e}, x_{e}, \tilde{x}$

$A, \Delta A ; C, \Delta C$

$Z_{2}-Z_{1} \in \mathbb{N}^{r}, Z_{2}-Z_{1} \in \mathbb{P}^{r}, Z_{1}, Z_{2} \in \mathbb{S}^{r}$.

positive integers; $n+n_{e}$.

$n, l, \hat{l}, q, n_{\mathrm{e}}, \tilde{n}$-dimensional vectors.

$\hat{C}, L, R$

$n \times n$ matrices; $l \times n$ matrices.

$A_{\mathrm{e}}, B_{\mathrm{e}}, C_{\mathrm{e}}, D_{\mathrm{e}}$

$\tilde{A}, \Delta \tilde{A}$

$\hat{l} \times n$ matrix, $q \times n$ matrix, $q \times q$ matrix, $R \in \mathbb{P}^{q}$.

$\tilde{A}, \Delta \tilde{A}$

$\tilde{R}$

$n_{\mathrm{e}} \times n_{\mathrm{e}}, n_{\mathrm{e}} \times l, q \times n_{\mathrm{e}}, q \times \hat{l}$ matrices.

$\tilde{R}$

$w_{0}(\cdot), w_{1}(\cdot)$

$V_{0}, V_{1}$

$V_{01}$

$\tilde{w}(\cdot), \tilde{V}$

$n, l$-dimensional white noise.

intensity of $w_{0}(\cdot), w_{1}(\cdot) ; V_{0} \in \mathbb{N}^{n}, V_{1} \in \mathbb{P}^{\prime}$.

$n \times l$ cross intensity of $w_{0}(\cdot), w_{1}(\cdot)$.

$\left[\begin{array}{c}w_{0}(\cdot) \\ B_{\mathrm{e}} w_{1}(\cdot)\end{array}\right],\left[\begin{array}{cc}V_{0} & V_{01} B_{\mathrm{e}}^{\mathrm{T}} \\ B_{\mathrm{e}} V_{01}^{\mathrm{T}} & B_{\mathrm{e}} V_{1} B_{\mathrm{e}}^{\mathrm{T}}\end{array}\right]$.

\section{Robust estimation problem}

Let $\mathscr{U} \subset \mathbb{R}^{n \times n} \times \mathbb{R}^{1 \times n}$ denote the set of uncertain perturbations $(\Delta A, \Delta C)$ of the nominal plant matrices $A$ and $C$.

Robust Estimation Problem. For fixed $n_{\mathrm{e}} \leq n$, determine $\left(A_{\mathrm{e}}, B_{\mathrm{e}}, C_{\mathrm{e}}, D_{\mathrm{e}}\right)$ such that, for the system consisting of the $n$-th-order disturbed plant

$$
\dot{x}(t)=(A+\Delta A) x(t)+w_{0}(t), \quad t \in[0, \infty),
$$

noisy and nonnoisy measurements

$$
\begin{aligned}
& y(t)=(C+\Delta C) x(t)+w_{1}(t), \\
& \hat{y}(t)=\hat{C} x(t),
\end{aligned}
$$

and $n_{\mathrm{e}}$-th-order nonstrictly proper state estimator

$$
\begin{aligned}
& \dot{x}_{\mathrm{e}}(t)=A_{\mathrm{e}} x_{\mathrm{e}}(t)+B_{\mathrm{e}} y(t), \\
& y_{\mathrm{e}}(t)=C_{\mathrm{e}} x_{\mathrm{e}}(t)+D_{\mathrm{e}} \hat{y}(t),
\end{aligned}
$$

the state-estimation error criterion

$$
J\left(A_{\mathrm{e}}, B_{\mathrm{e}}, C_{\mathrm{e}}, D_{\mathrm{e}}\right) \triangleq \sup _{(\Delta A, \Delta C) \subseteq \mathscr{L}} \limsup _{t \rightarrow \infty} \mathbb{E}\left[L x(t)-y_{\mathrm{e}}(t)\right]^{\mathrm{T}} R\left[L x(t)-y_{\mathrm{e}}(t)\right]
$$

is minimized.

Note that the augmented systen (3.1)-(3.5) can be written as

$$
\dot{\tilde{x}}(t)=(\tilde{A}+\Delta \tilde{A}) \tilde{x}(t)+\tilde{w}(t), \quad t \in[0, \infty),
$$

where $\tilde{x}(t) \triangleq\left[x^{\mathrm{T}}(t), x_{\mathrm{e}}^{\mathrm{T}}(t)\right]^{\mathrm{T}}$. The cost can be expressed in terms of the augmented second-moment matrix. 
Proposition 3.1. For given $\left(A_{\mathrm{e}}, B_{\mathrm{e}}, C_{\mathrm{e}}, D_{\mathrm{e}}\right)$ and $(\Delta A, \Delta C) \in \mathscr{U}$ the second-moment matrix

$$
\tilde{Q}_{\Delta \tilde{A}}(t) \triangleq \mathbb{E}\left[\tilde{x}(t) \tilde{x}^{\mathrm{T}}(t)\right], \quad t \in[0, \infty),
$$

satisfies

$$
\dot{\tilde{Q}}_{\Delta \tilde{A}}(t)=(\tilde{A}+\Delta \tilde{A}) \tilde{Q}_{\Delta \tilde{A}}(t)+\tilde{Q}_{\Delta \tilde{A}}(t)(\tilde{A}+\Delta \tilde{A})^{\mathrm{T}}+\tilde{V}, \quad t \in[0, \infty) .
$$

Furthermore,

$$
J\left(A_{\mathrm{e}}, B_{\mathrm{e}}, C_{\mathrm{e}}, D_{\mathrm{e}}\right)=\sup _{(\Delta A, \Delta C) \in \mathscr{Q}} \limsup _{t \rightarrow \infty} \operatorname{tr} \tilde{Q}_{\Delta \tilde{A}}(t) \tilde{R} .
$$

\section{Sufficient conditions for robust performance}

Lemma 4.1. Suppose the system (3.7) is stable for all $(\Delta A, \Delta C) \in \mathscr{U}$. Then

$$
J\left(A_{\mathrm{e}}, B_{\mathrm{e}}, C_{\mathrm{e}}, D_{\mathrm{e}}\right)=\sup _{(\Delta A, \Delta C) \in \mathscr{Q}} \operatorname{tr} \tilde{Q}_{\Delta \tilde{A}} \tilde{R},
$$

where $\tilde{Q}_{\Delta \tilde{A}} \in \mathbb{N}^{\bar{n}}$ is the unique solution to

$$
0=(\tilde{A}+\Delta \tilde{A}) \tilde{Q}_{\Delta \tilde{A}}+\tilde{Q}_{\Delta \tilde{A}}(\tilde{A}+\Delta \tilde{A})^{\mathrm{T}}+\tilde{V} .
$$

We now seek upper bounds for $J\left(A_{\mathrm{e}}, B_{\mathrm{e}}, C_{\mathrm{e}}, D_{\mathrm{e}}\right)$.

Theorem 4.1. Let $\Omega: \mathbb{N}^{\bar{n}} \times \mathbb{R}^{n_{\mathrm{e}} \times 1} \rightarrow \mathbb{S}^{\bar{n}}$ be such that

$$
\Delta \tilde{A} \mathscr{Q}+\mathscr{Q} \Delta \tilde{A}^{\mathrm{T}} \leq \Omega\left(\mathscr{Q}, B_{\mathrm{e}}\right), \quad(\Delta A, \Delta C) \in \mathscr{U}, \quad\left(\mathscr{Q}, B_{\mathrm{e}}\right) \in \mathbb{N}^{\bar{n}} \times \mathbb{R}^{n_{\mathrm{e}} \times l},
$$

and, for given $\left(A_{\mathrm{e}}, B_{\mathrm{e}}, C_{\mathrm{e}}, D_{\mathrm{e}}\right)$, suppose there exists $\mathscr{Q} \in \mathbb{N}^{n}$ satisfying

$$
0=\tilde{A} \mathscr{Q}+\mathscr{Q} \tilde{A}^{\mathrm{T}}+\Omega\left(\mathscr{Q}, B_{e}\right)+\tilde{V},
$$

and suppose the pair $\left(\tilde{V}^{1 / 2}, \tilde{A}+\Delta \tilde{A}\right)$ is detectable for all $(\Delta A, \Delta C) \in \mathscr{U}$. Then $A_{\mathrm{e}}$ is asymptotically stable, $A+\Delta A$ is asymptotically stable for all $(\Delta A, \Delta C) \in \mathscr{U}$,

$$
\tilde{Q}_{\Delta \bar{A}} \leq \mathscr{Q}, \quad(\Delta A, \Delta C) \in \mathscr{U},
$$

where $\tilde{Q}_{\Delta \tilde{A}}$ satisfies $(4.2)$, and

$$
J\left(A_{\mathrm{e}}, B_{\mathrm{e}}, C_{\mathrm{e}}, D_{\mathrm{e}}\right) \leq \operatorname{tr} \mathscr{Q} \tilde{R}
$$

Proof. For all $(\Delta A, \Delta C) \in \mathscr{U},(4.4)$ is equivalent to

$$
0=(\tilde{A}+\Delta \tilde{A}) \mathscr{2}+\mathscr{2}(\tilde{A}+\Delta \tilde{A})^{\mathrm{T}}+\Psi\left(\mathscr{Q}, B_{\mathrm{e}}, \Delta \tilde{A}\right)+\tilde{V}
$$

where

$$
\Psi\left(\mathscr{Q}, B_{\mathrm{e}}, \Delta \tilde{A}\right) \triangleq \Omega\left(\mathscr{Q}, B_{\mathrm{e}}\right)-\left(\tilde{\Delta} A \mathscr{Q}+\mathscr{Q} \Delta \tilde{A}^{\mathrm{T}}\right) .
$$

Note that by $(4.3), \Psi\left(\mathscr{Q}, B_{\mathrm{e}}, \Delta \tilde{A}\right) \geq 0$ for all $(\Delta A, \Delta C) \in \mathscr{U}$. Since $\left(\tilde{V}^{1 / 2}, \tilde{A}+\Delta \tilde{A}\right)$ is detectable for all $(\Delta A, \Delta C) \in \mathscr{U}$, it follows from Theorem 3.6 of [17] that $\left(\left(\tilde{V}+\Psi\left(\mathscr{2}, B_{\mathrm{e}}, \Delta \tilde{A}\right)\right)^{1 / 2}, \tilde{A}+\Delta \tilde{A}\right)$ is detectable for all $(\Delta A, \Delta C) \in \mathscr{U}$. Hence Lemma 12.2 of [17] implies $\tilde{A}+\Delta \tilde{A}$ is asymptotically stable for all $(\Delta A, \Delta C) \in \mathscr{U}$. Since $\tilde{A}+\Delta \tilde{A}$ is lower block triangular, $A_{\mathrm{e}}$ is asymptotically stable and $A+\Delta A$ is asymptotically stable for all $(\Delta A, \Delta C) \in \mathscr{U}$. Next, substracting (4.2) from (4.7) yields

$$
0=(\tilde{A}+\Delta \tilde{A})\left(\mathscr{Q}-\tilde{Q}_{\Delta \tilde{A}}\right)+\left(\mathscr{Q}-\tilde{Q}_{\Delta \tilde{A}}\right)(\tilde{A}+\Delta \tilde{A})^{\mathrm{T}}+\Psi\left(\mathscr{Q}, B_{\mathrm{e}}, \Delta \tilde{A}\right),
$$


or, equivalently, (since $\tilde{A}+\Delta \tilde{A}$ is asymptotically stable)

$$
\mathscr{Q}-\tilde{Q}_{\Delta \tilde{A}}=\int_{0}^{\infty} \mathrm{e}^{(\tilde{A}+\Delta \tilde{A}) t} \Psi\left(\mathscr{Q}, B_{\mathrm{e}}, \Delta \tilde{A}\right) \mathrm{e}^{(\tilde{A}+\Delta \tilde{A})^{\mathrm{T}} t} \mathrm{~d} t \geq 0,
$$

which implies (4.5). Finally, (4.5) and (4.1) yield (4.6).

\section{Uncertainty structure}

The uncertainty set $\mathscr{U}$ is assumed to be of the form

$$
\begin{aligned}
\mathscr{U}=\left\{(\Delta A, \Delta C) \in \mathbb{R}^{n \times n} \times \mathbb{R}^{l \times n}: \Delta A=\sum_{i=1}^{p} D_{i} M_{i} N_{i} E_{i},\right. \\
\left.\quad \Delta C=\sum_{i=1}^{p} F_{i} M_{i} N_{i} E_{i}, M_{i} M_{i}^{\mathrm{T}} \leq \bar{M}_{i}, N_{i}^{\mathrm{T}} N_{i} \leq \bar{N}_{i}, i=1, \ldots, p\right\},
\end{aligned}
$$

where, for $i=1, \ldots, p, D_{i} \in \mathbb{R}^{n \times r_{i}}, E_{i} \in \mathbb{R}^{t_{i} \times n}$ and $F_{i} \in \mathbb{R}^{1 \times r_{i}}$ are fixed matrices denoting the structure of the uncertainty; $\bar{M}_{i} \in \mathbb{N}^{r_{i}}$ and $\bar{N}_{i} \in \mathbb{N}^{t_{i}}$ are given uncertainty bounds; and $M_{i} \in \mathbb{R}^{r_{i} \times s_{i}}, N_{i} \in \mathbb{R}^{s_{i} \times t_{i}}$ are uncertain matrices. The closed-loop system thus has structured uncertainty of the form

$$
\Delta \tilde{A}=\sum_{i=1}^{p} \tilde{D}_{i} M_{i} N_{i} \tilde{E}_{i}
$$

where

$$
\tilde{D}_{i} \triangleq\left[\begin{array}{c}
D_{i} \\
B_{e} F_{i}
\end{array}\right], \quad \tilde{E}_{i} \triangleq\left[\begin{array}{ll}
E_{i} & 0
\end{array}\right] .
$$

The special case $\bar{M}_{i}=\mu_{i}^{2} I_{r_{i}}, \bar{N}_{i}=\nu_{i}^{2} I_{t i}$ is worth noting.

Proposition 5.1. Let $\mu_{i}, \quad \nu_{i} \geq 0, i=1, \ldots, p$. Then $M_{i} M_{i}^{\mathrm{T}} \leq \mu_{i}^{2} I_{r_{i}}$ and $N_{i}^{\mathrm{T}} N_{i} \leq \nu_{i}^{2} I_{i_{i}}$ if and only if $\sigma_{\max }\left(M_{i}\right) \leq \mu_{i}$ and $\sigma_{\max }\left(N_{i}\right) \leq \nu_{i}$.

Remark 5.1. $\mathscr{U}$ given by (5.1) is directly related to the structured stability radius introduced in [10]. Setting $p=1, \bar{M}_{1}=\mu_{1} I_{r_{1}}, r_{1}=s_{1}, \bar{N}_{1}=I_{r_{1}}$ and $N_{1} \equiv I_{r_{1}}$ yields the setting of [10]. For a similar formulation, see [13].

\section{The Petersen-Hollot bound}

Given $\mathscr{U}$ as defined in (5.1), we now specify $\Omega$ 'satisfying (4.3).

Proposition 6.1. The bound $\Omega$ given by

$$
\Omega\left(\mathscr{Q}, B_{\mathrm{e}}\right) \triangleq \sum_{i=1}^{p} \tilde{D}_{i} \bar{M}_{i} \tilde{D}_{i}^{\mathrm{T}}+\mathscr{Q} \tilde{E}_{i}^{\mathrm{T}} \bar{N}_{i} \tilde{E}_{i} \mathscr{Q}
$$

satisfies (4.3) with $\mathscr{U}$ given by (5.1). 
Proof. For $i=1, \ldots, p$,

$$
\begin{aligned}
0 & \leq\left[\tilde{D}_{i} M_{i}-\mathscr{Q} \tilde{E}_{i}^{\mathrm{T}} N_{i}^{\mathrm{T}}\right]\left[\tilde{D}_{i} M_{i}-\mathscr{Q} \tilde{E}_{i}^{\mathrm{T}} N_{i}^{\mathrm{T}}\right]^{\mathrm{T}} \\
& =\tilde{D}_{i} M_{i} M_{i}^{\mathrm{T}} \tilde{D}_{i}^{\mathrm{T}}+\mathscr{Q} \tilde{E}_{i}^{\mathrm{T}} N_{i}^{\mathrm{T}} N_{i} \tilde{E}_{i} \mathscr{Q}-\left(\tilde{D}_{i} M_{i} N_{i} \tilde{E}_{i} \mathscr{Q}+\mathscr{Q} \tilde{E}_{i}^{\mathrm{T}} N_{i}^{\mathrm{T}} M_{i}^{\mathrm{T}} \tilde{D}_{i}^{\mathrm{T}}\right) \\
& \leq \tilde{D}_{i} \bar{M}_{i} \tilde{D}_{i}^{\mathrm{T}}+\mathscr{Q} \tilde{E}_{i}^{\mathrm{T}} \bar{N}_{i} \tilde{E}_{i} \mathscr{Q}-\left(\tilde{D}_{i} M_{i} N_{i} \tilde{E}_{i} \mathscr{Q}+\mathscr{Q} \tilde{E}_{i}^{\mathrm{T}} N_{i}^{\mathrm{T}} M_{i}^{\mathrm{T}} \tilde{D}_{i}^{\mathrm{T}}\right) .
\end{aligned}
$$

Summing over $i$ yields (4.3).

Remark 6.1. The bound (6.1) is used in [12] for unit-rank perturbations while a more general treatment appears in [13].

\section{The auxiliary minimization problem}

Our goal is to minimize the error bound (4.6).

Auxiliary Minimization Problem. Determine $\left(\mathscr{Q}, A_{\mathrm{e}}, B_{\mathrm{e}}, C_{\mathrm{e}}, D_{\mathrm{e}}\right)$ with $\mathscr{2} \in \mathbb{N}^{\tilde{n}}$ which minimizes

$$
\mathscr{J}\left(\mathscr{Q}, A_{\mathrm{e}}, B_{\mathrm{e}}, C_{\mathrm{e}}, D_{\mathrm{e}}\right) \triangleq \operatorname{tr} \mathscr{Q} \tilde{R}
$$

subject to

$$
0=\tilde{A} \mathscr{Q}+\mathscr{Q} \tilde{A}^{\mathrm{T}}+\sum_{i=1}^{p}\left[\tilde{D}_{i} \bar{M}_{i} \tilde{D}_{i}^{\mathrm{T}}+\mathscr{Q} \tilde{E}_{i}^{\mathrm{T}} \bar{N}_{i} \tilde{E}_{i} \mathscr{Q}\right]+\tilde{V},
$$

and

$$
\left(\tilde{V}^{1 / 2}, \tilde{A}+\Delta \tilde{A}\right) \text { is detectable, }(\Delta A, \Delta C) \in \mathscr{U} .
$$

Proposition 7.1. If $\left(\mathscr{Q}, A_{\mathrm{e}}, B_{\mathrm{e}}, C_{\mathrm{e}}, D_{\mathrm{e}}\right)$ satisfies (7.2) and (7.3) with $\mathscr{Q} \geq 0$, then $\tilde{A}+\Delta \tilde{A}$ is asymptotically stable for all $(\Delta A, \Delta C) \in \mathscr{U}$, and

$$
J\left(A_{\mathrm{e}}, B_{\mathrm{e}}, C_{\mathrm{e}}, D_{\mathrm{e}}\right) \leq \mathscr{J}\left(\mathscr{Q}, A_{\mathrm{e}}, B_{\mathrm{e}}, C_{\mathrm{e}}, D_{\mathrm{e}}\right) .
$$

Proof. With $\Omega$ given by (6.1), (7.2) is equivalent to (4.4). Hence, with (7.3), the hypotheses of Theorem 4.1 are satisfied so that the augmented system is stable over $\mathscr{U}$ with estimation bound (4.6). Note that with (7.1), (7.4) is merely a restatement of (4.6).

\section{Necessary conditions for the auxiliary minimization problem}

Rigorous application of the Lagrange multiplier technique requires additional technical assumptions. Specifically, we further restrict $\left(\mathcal{Q}, A_{\mathrm{e}}, B_{\mathrm{e}}, C_{\mathrm{e}}, D_{\mathrm{e}}\right)$ to the set

$$
\mathscr{S} \triangleq\left\{\left(\mathscr{Q}, A_{\mathrm{e}}, B_{\mathrm{e}}, C_{\mathrm{e}}, D_{\mathrm{e}}\right): \mathscr{Q} \in \mathbb{P}^{\bar{n}}, \tilde{\mathscr{A}} \text { is asymptotically stable, }\left(A_{\mathrm{e}}, B_{\mathrm{e}}, C_{\mathrm{e}}\right)\right. \text { is controllable }
$$

$$
\text { and observable, and } \left.\hat{C}\left(Q_{1}-Q_{12} Q_{2}^{-1} Q_{12}^{\mathrm{T}}\right) \hat{C}^{\mathrm{T}}>0\right\} \text {, }
$$

where $(\oplus$ denotes Kronecker sum [3])

$$
\tilde{\mathscr{A}} \triangleq\left(\tilde{A}+\sum_{i=1}^{p} \tilde{E}_{i}^{\mathrm{T}} \bar{N}_{i} \tilde{E}_{i} \mathscr{Q}\right) \oplus\left(\tilde{A}+\sum_{i=1}^{p} \tilde{E}_{i}^{\mathrm{T}} \bar{N}_{i} \tilde{E}_{i} \mathscr{Q}\right)
$$

and $\mathscr{Q}$ is partitioned as in Appendix $\mathrm{A}$. As shown in Appendix $\mathrm{A}, Q_{2}$ is invertible since $\left(A_{\mathrm{e}}, B_{\mathrm{e}}\right)$ is controllable. The positive definiteness condition holds when $\hat{C}$ has full row rank and $\mathscr{Q}$ is positive definite. 
As can be seen from the proof of Theorem 8.1 in Appendix A, this condition implies the existence of the projection $\tau_{1}$ defined below. Note that $\mathscr{S}$ is open.

Remark 8.1. The constraint $\left(\mathscr{Q}, A_{\mathrm{e}}, B_{\mathrm{e}}, C_{\mathrm{e}}, D_{\mathrm{e}}\right) \in \mathscr{S}$ is not required for robust estimation. As will be seen from the proof of Theorem 8.1, the set $\mathscr{S}$ constitutes sufficient conditions under which the Lagrange multiplier technique is applicable to the Auxiliary Minimization Problem. Specifically, asymptotic stability of $\tilde{\mathscr{A}}$ serves as a normality condition which further implies that the dual $\mathscr{P}$ of $\mathscr{Q}$ satisfying (A.2) is nonnegative definite. Furthermore, $\left(A_{\mathrm{e}}, B_{\mathrm{e}}, C_{\mathrm{e}}\right)$ minimal is a nondegeneracy condition which implies that the lower right $n_{\mathrm{e}} \times n_{\mathrm{e}}$ subblocks of $\mathscr{Q}$ and $\mathscr{P}$ are positive definite. It is extremely important to emphasize that Proposition 7.1 shows that it is not necessary for guaranteed robust estimation that an admissible quadruple obtained by solving the necessary conditions actually be shown to be an element of $\mathscr{S}$.

For arbitrary $Q \in \mathbb{R}^{n \times n}$ define the following notation:

$$
\begin{aligned}
& V_{1 \mathrm{a}} \triangleq V_{1}+\sum_{i=1}^{p} F_{i} \bar{M}_{i} F_{i}^{\mathrm{T}}, \quad Q_{\mathrm{a}} \triangleq V_{01}+Q C^{\mathrm{T}}+\sum_{i=1}^{p} D_{i} \bar{M}_{i} F_{i}^{\mathrm{T}}, \\
& D \triangleq \sum_{i=1}^{p} D_{i} \bar{M}_{i} D_{i}^{\mathrm{T}}, \quad E \triangleq \sum_{i=1}^{p} E_{i}^{\mathrm{T}} \bar{N}_{i} E_{i}, \quad A_{Q} \triangleq A-Q_{\mathrm{a}} V_{1 \mathrm{a}}^{-1} C .
\end{aligned}
$$

The following factorization lemma is needed for the statement of the main result. See [1] for details.

Lemma 8.1. If $\hat{Q}, \hat{P} \in \mathbb{N}^{n}$ and $\operatorname{rank} \hat{Q} \hat{P}=n_{\mathrm{e}}$, then there exist $n_{\mathrm{e}} \times n G, \Gamma$ and $n_{\mathrm{e}} \times n_{\mathrm{e}}$ invertible $M$ such that

$$
\hat{Q} \hat{P}=G^{\mathrm{T}} M \Gamma, \quad \Gamma G^{\mathrm{T}}=I_{n_{\mathrm{e}}} .
$$

Furthermore, $G, M$ and $\Gamma$ are unique except for a change of basis in $\mathbb{R}^{n} \mathrm{c}$.

Since $\hat{Q} \hat{P}$ is diagonalizable it has a group generalized inverse $(\hat{Q} \hat{P})^{\#}=G^{\mathrm{T}} M^{-1} \Gamma$ and

$$
\tau \triangleq \hat{Q} \hat{P}(\hat{Q} \hat{P})^{\#}=G^{\mathrm{T}} \Gamma
$$

is an oblique projection. Define the complementary projection $\tau_{\perp} \triangleq I_{n}-\tau$ and call $(G, M, \Gamma)$ satisfying (8.1) and (8.2) a projective factorization of $\hat{Q} \hat{P}$.

Theorem 8.1. ( $\left.\mathscr{Q}, A_{\mathrm{e}}, B_{\mathrm{e}}, C_{\mathrm{e}}, D_{\mathrm{e}}\right) \in \mathscr{S}$ is an extremal of the Auxiliary Minimization problem with $\mathscr{U}$ given by (5.1) if and only if there exist $Q, \hat{Q}, \hat{P} \in \mathbb{N}^{n}$ such that $\mathscr{Q}, A_{\mathrm{e}}, B_{\mathrm{e}}, C_{\mathrm{e}}, D_{\mathrm{e}}$ are given by

$$
\begin{aligned}
& \mathscr{Q}=\left[\begin{array}{cc}
Q+\hat{Q} & \hat{Q} \Gamma^{\mathrm{T}} \\
\Gamma \hat{Q} & \Gamma \hat{Q} \Gamma^{\mathrm{T}}
\end{array}\right], \\
& A_{\mathrm{e}}=\Gamma\left(A-Q_{\mathrm{a}} V_{1 \mathrm{a}}^{-1} C+Q E\right) G^{\mathrm{T}}, \\
& B_{\mathrm{e}}=\Gamma Q_{\mathrm{a}} V_{1 \mathrm{a}}^{-1}, \\
& C_{\mathrm{e}}=L \tau_{1 \perp} G^{\mathrm{T}}, \\
& D_{\mathrm{e}}=L Q \hat{C}^{\mathrm{T}}\left(\hat{C} Q \hat{C}^{\mathrm{T}}\right)^{-1},
\end{aligned}
$$

for some projective factorization $(G, M, \Gamma)$ of $\hat{Q} \hat{P}$, and such that $Q, \hat{Q}, \hat{P}$ satisfy

$$
\begin{aligned}
& 0=A Q+Q A^{\mathrm{T}}+V_{0}+D+Q E Q-Q_{\mathrm{a}} V_{1 \mathrm{a}}^{-1} Q_{\mathrm{a}}^{\mathrm{T}}+\tau_{\perp} Q_{\mathrm{a}} V_{1 \mathrm{a}}^{-1} Q_{\mathrm{a}}^{\mathrm{T}} \tau_{\perp}^{\mathrm{T}}, \\
& 0=(A+Q E) \hat{Q}+\hat{Q}(A+Q E)^{\mathrm{T}}+\hat{Q} E \hat{Q}+Q_{\mathrm{a}} V_{1 \mathrm{a}}^{-1} Q_{\mathrm{a}}^{\mathrm{T}}-\tau_{\perp} Q_{\mathrm{a}} V_{1 a}^{-1} Q_{\mathrm{a}}^{\mathrm{T}} \tau_{\perp}^{\mathrm{T}}, \\
& 0=\left(A_{Q}+Q E\right)^{\mathrm{T}} \hat{P}+\hat{P}\left(A_{Q}+Q E\right)+\tau_{1 \perp}^{\mathrm{T}} L^{\mathrm{T}} R L \tau_{1 \perp}-\tau_{\perp}^{\mathrm{T}} \tau_{1 \perp}^{\mathrm{T}} L^{\mathrm{T}} R L \tau_{1 \perp} \tau_{\perp}, \\
& \operatorname{rank} \hat{Q}=\operatorname{rank} \hat{P}=\operatorname{rank} \hat{Q} \hat{P}=n_{\mathrm{e}},
\end{aligned}
$$


where

$$
\tau_{1} \triangleq Q \hat{C}^{\mathrm{T}}\left(\hat{C} Q \hat{C}^{\mathrm{T}}\right)^{-1} \hat{C}, \quad \tau_{1 \perp} \triangleq I_{n}-\tau_{1} .
$$

Theorem 8.1 (proved in Appendix A) presents necessary conditions for the Auxiliary Minimization Problem which explicitly characterize extremals $\left(2, A_{\mathrm{e}}, B_{\mathrm{e}}, C_{\mathrm{e}}, D_{\mathrm{e}}\right)$. These necessary conditions consist of a system of two modified Lyapunov equations and one modified Riccati equation coupled by two oblique projections $\tau$ and $\tau_{1}$ and uncertainty terms. The projections $\tau$ and $\tau_{1}$ correspond to reduced estimator order and singular observation noise, respectively.

Several special cases can immediately be discerned. For example, in the full-order estimator case $n_{\mathrm{e}}=n$, set $\tau=I_{n}$ so that $\tau_{\perp}=0$. Now the last term in each of (8.9)-(8.11) can be deleted and $G$ and $\Gamma$ in (8.4)-(8.7) can be taken to be the identity. Furthermore, since $\hat{Q}$ and $\hat{P}$ now play no role in determining the optimal estimator, equations (8.10) and (8.11) are superfluous. If, furthermore, $D_{i}, E_{i}$ and $F_{i}$ are zero, then (8.9) reduces to the standard observer Riccati equation of steady-state Kalman filter theory. Alternatively, the case in which the static estimator gain $D_{\mathrm{e}}$ is absent can be handled by ignoring (8.8) and setting $\tau_{1}=0$. If, furthermore, the uncertainty terms are deleted then the results of [1] are recovered.

\section{Sufficient conditions for robust, reduced-order estimation}

The main result guaranteeing robust estimation can now be stated.

Theorem 9.1. Suppose there exist $Q, \hat{Q}, \hat{P} \in \mathbb{N}^{n}$ satisfying (8.9)-(8.12), let $A_{\mathrm{e}}, B_{\mathrm{e}}, C_{\mathrm{e}}, D_{\mathrm{e}}$ be given by (8.5)-(8.8), and suppose that $\left(\tilde{V}^{1 / 2}, \tilde{A}+\Delta \tilde{A}\right)$ is detectable for all $(\Delta A, \Delta C) \in \mathscr{U}$ with $\mathscr{U}$ given by (5.1). Then $A_{\mathrm{e}}$ is asymptotically stable, $A+\Delta A$ is asymptotically stable for all $(\Delta A, \Delta C) \in \mathscr{U}$, and the estimation error satisfies the bound

$$
J\left(A_{\mathrm{e}}, B_{\mathrm{e}}, C_{\mathrm{e}}, D_{\mathrm{e}}\right) \leq \operatorname{tr} Q \tau_{1 \perp}^{\mathrm{T}} L^{\mathrm{T}} R L \tau_{1 \perp} .
$$

Proof. Theorem 8.1 implies 2 given by (8.4) satisfies (7.2). With the detectability assumption the result follows from Proposition 7.1.

Remark 9.1. Note that if $\hat{C}=L$ then $C_{\mathrm{e}}=0$ and the estimation bound (9.1) is zero since $\hat{\mathrm{C}} \tau_{1 \perp}=0$. This is, of course, to be expected since perfect estimation is achievable in this case.

Remark 9.2. The problem of designing reduced-order, robust estimators for unstable systems remains an area for future research.

\section{Appendix A: Proof of Theorem 8.1}

Partition $\tilde{n} \times \tilde{n} \mathscr{Q}, \mathscr{P}$ into $n \times n, n \times n_{\mathrm{e}}$, and $n_{\mathrm{e}} \times n_{\mathrm{e}}$ subblocks as

$$
\mathscr{Q}=\left[\begin{array}{ll}
Q_{1} & Q_{12} \\
Q_{12}^{T} & Q_{2}
\end{array}\right], \quad \mathscr{P}=\left[\begin{array}{cc}
P_{1} & P_{12} \\
P_{12}^{T} & P_{2}
\end{array}\right],
$$

and define the $n \times n$ nonnegative-definite matrices

$$
\begin{array}{ll}
Q \triangleq Q_{1}-Q_{12} Q_{2}^{-1} Q_{12}^{\mathrm{T}}, & P \triangleq P_{1}-P_{12} P_{2}^{-1} P_{12}^{\mathrm{T}}, \\
\hat{Q} \triangleq Q_{12} Q_{2}^{-1} Q_{12}^{\mathrm{T}}, & \hat{P} \triangleq P_{12} P_{2}^{-1} P_{12}^{\mathrm{T}},
\end{array}
$$

and the $n_{\mathrm{e}} \times n, n_{\mathrm{e}} \times n_{\mathrm{e}}, n_{\mathrm{e}} \times n$ matrices

$$
G \triangleq Q_{2}^{-1} Q_{12}^{\mathrm{T}}, \quad M \triangleq Q_{2} P_{2}, \quad \Gamma \triangleq-P_{2}^{-1} P_{12}^{\mathrm{T}} .
$$

The existence of $Q_{2}^{-1}$ and $P_{2}^{-1}$ is shown below. 
To optimize (7.1) over the open set $\mathscr{S}^{\prime}$, where $\mathscr{S}^{\prime} \triangleq\left\{\left(\mathscr{Q}, A_{\mathrm{e}}, B_{\mathrm{e}}, C_{\mathrm{e}}, D_{\mathrm{c}}\right) \in \mathscr{S}:\right.$ (7.3) is satisfied $\}$, subject to the constraint (7.2), form the Lagrangian

$$
\mathscr{L}\left(\mathscr{Q}, A_{\mathrm{e}}, B_{\mathrm{e}}, C_{\mathrm{e}}, D_{\mathrm{e}}\right) \triangleq \operatorname{tr}\left[\lambda \mathscr{Q} \tilde{R}+\left(\tilde{A} \mathscr{Q}+\mathscr{Q} \tilde{A}^{\mathrm{\top}}+\sum_{i=1}^{p} \tilde{D}_{i} \bar{M}_{i} \tilde{D}_{i}^{\mathrm{T}}+\mathscr{Q} \tilde{E}_{i}^{\mathrm{\top}} \bar{N}_{i} \tilde{E}_{i} \mathscr{Q}+\tilde{V}\right) \mathscr{P}\right],
$$

where the multipliers $\lambda \geq 0$ and $\mathscr{P} \in \mathbb{R}^{\tilde{n} \times \tilde{n}}$ are not both zero. We thus obtain

$$
\frac{\partial \mathscr{L}}{\partial \mathscr{Q}}=\tilde{A}^{\mathrm{T}} \mathscr{P P}+\mathscr{P} \tilde{A}+\sum_{i=1}^{p} \tilde{E}_{i}^{\mathrm{T}} \bar{N}_{i} \tilde{E}_{i} \mathscr{Q P P}+\mathscr{P} \mathscr{Q} \tilde{E}_{i}^{\mathrm{T}} \bar{N}_{i} \tilde{E}_{i}+\lambda \tilde{R}
$$

Setting $\partial \mathscr{L} / \partial \mathscr{Q}=0$ yields ('vec' is defined in [3])

$\tilde{\mathscr{A}}^{\mathrm{T}}$ vec $\mathscr{P}=-\lambda$ vec $\tilde{R}$.

Since $\tilde{A}$ is assumed to be invertible, $\lambda=0$ implies $\mathscr{P}=0$. Hence, without loss of generality, set $\lambda=1$. Since, furthermore, $\tilde{A}$ is assumed to be asymptotically stable, $\mathscr{P}$ is nonnegative definite. The stationarity conditions are given by

$$
\begin{aligned}
& \frac{\partial \mathscr{L}}{\partial \mathscr{P}}=\tilde{A} \mathscr{Q}+\mathscr{Q} \tilde{A}^{\mathrm{T}}+\sum_{i=1}^{p} \tilde{D}_{i} \bar{M}_{i} \tilde{D}_{i}^{\mathrm{T}}+\mathscr{Q} \tilde{E}_{i}^{\mathrm{T}} \bar{N}_{i} \tilde{E}_{i} \mathscr{Q}+\tilde{V}=0, \\
& \frac{\partial \mathscr{L}}{\partial \mathscr{Q}}=\tilde{A}^{\mathrm{T}} \mathscr{P}+\mathscr{P} \tilde{A}+\sum_{i=1}^{p} \tilde{E}_{i}^{\mathrm{T}} \bar{N}_{i} \tilde{E}_{i} \mathscr{Q P}+\mathscr{P} \mathscr{Q} \tilde{E}_{i}^{\mathrm{T}} \bar{N}_{i} \tilde{E}_{i}+\tilde{R}=0, \\
& \frac{\partial \mathscr{L}}{\partial A_{\mathrm{e}}}=P_{12}^{\mathrm{T}} Q_{12}+P_{2} Q_{2}=0, \\
& \frac{\partial \mathscr{L}}{\partial B_{\mathrm{e}}}=P_{12}^{\mathrm{T}} V_{01}+\left(P_{12}^{\mathrm{T}} Q_{1}+P_{2} Q_{12}^{\mathrm{T}}\right) C^{\mathrm{T}}+P_{2} B_{\mathrm{e}} V_{1 \mathrm{a}}=0, \\
& \frac{\partial \mathscr{L}}{\partial C_{\mathrm{e}}}=-R L Q_{12}+R D_{\mathrm{e}} C Q_{12}+R C_{\mathrm{e}} Q_{2}=0, \\
& \frac{\partial \mathscr{L}}{\partial D_{\mathrm{e}}}=-R L Q_{1} \hat{C}^{\mathrm{T}}+R D_{\mathrm{e}} \hat{C} Q_{1} \hat{C^{\mathrm{T}}}+R C_{\mathrm{e}} Q_{12}^{\mathrm{T}} \hat{C}^{\mathrm{T}}=0 .
\end{aligned}
$$

Expanding (A.1) and (A.2) yields

$$
\begin{aligned}
& 0=A Q_{1}+Q_{1} A^{\mathrm{T}}+D+Q_{1} E Q_{1}+V_{0}, \\
& 0=A Q_{12}+Q_{1} C^{\mathrm{T}} B_{\mathrm{e}}^{\mathrm{T}}+Q_{12} A_{\mathrm{e}}^{\mathrm{T}}+Q_{1} E Q_{12}+V_{01} B_{\mathrm{e}}^{\mathrm{T}}, \\
& 0=B_{\mathrm{e}} C Q_{12}+A_{\mathrm{e}} Q_{2}+Q_{12}^{\mathrm{T}} C^{\mathrm{T}} B_{\mathrm{e}}^{\mathrm{T}}+Q_{2} A_{\mathrm{e}}^{\mathrm{T}}+B_{\mathrm{e}} V_{1 \mathrm{a}} B_{\mathrm{e}}^{\mathrm{T}}+Q_{12}^{\mathrm{T}} E Q_{12}, \\
& 0=P_{12} A_{\mathrm{e}}+A^{\mathrm{T}} P_{12}+C^{\mathrm{T}} B_{\mathrm{e}}^{\mathrm{T}} P_{2}+E\left(P_{12}^{\mathrm{T}} Q_{1}+P_{2} Q_{12}^{\mathrm{T}}\right)^{\mathrm{T}}-L^{\mathrm{T}} R C_{\mathrm{e}}+C^{\mathrm{T}} D_{\mathrm{e}}^{\mathrm{T}} R C_{\mathrm{e}}, \\
& 0=P_{2} A_{\mathrm{e}}+A_{\mathrm{e}}^{\mathrm{T}} P_{2}+C_{\mathrm{e}}^{\mathrm{T}} R C_{\mathrm{e}} .
\end{aligned}
$$

Note that the $(1,1)$ subblock of equation (A.2) characterizing $P_{1}$ has been omitted from the above equations since the estimator gains are independent of $P_{1}$. Writing (A.9) as (see [1,9])

$$
0=\left(A_{\mathrm{e}}+B_{\mathrm{e}} C Q_{12} Q_{2}^{+}\right) Q_{2}+Q_{2}\left(A_{\mathrm{e}}+B_{\mathrm{e}} C Q_{12} Q_{2}^{+}\right)^{\mathrm{T}}+Q_{2}\left(Q_{12} Q_{2}^{+}\right)^{\mathrm{T}} E Q_{12} Q_{2}^{+} Q_{2}+B_{\mathrm{e}} V_{1 \mathrm{a}} B_{\mathrm{e}}^{\mathrm{T}}
$$

where $Q_{2}^{+}$is the Moore-Penrose or Drazin generalized inverse of $Q_{2}$, it follows from [17], Lemmas 2.1 and 12.2, that $Q_{2}$ is positive definite. Similarly, (A.11) implies that $P_{2}$ is positive definite.

Next (8.4), (8.6)-(8.8) follow from the definition of $2,(A .4)-(A .6)$ by using the identities

$$
Q_{1}=Q+\hat{Q}, \quad P_{1}=P+\hat{P}, \quad Q_{12}=\hat{Q} \Gamma^{\mathrm{T}}, \quad P_{12}=-\hat{P} G^{\mathrm{\top}}, \quad Q_{2}=\Gamma \hat{Q} \Gamma^{\mathrm{T}}, \quad P_{2}=G \hat{P} G^{\mathrm{T}} .
$$


Computing either $\Gamma$ (A.8)-(A.9) or $G(\mathrm{~A} .10)+$ (A.11) yields (8.5). Inserting (8.5)-(8.8) into (A.7)-(A.11) and using (A.7) $+G^{\mathrm{T}} \Gamma(\mathrm{A} .8)-(\mathrm{A} .8) G-(\mathrm{A} .8 G)^{\mathrm{T}}$ and $G^{\mathrm{T}} \Gamma(\mathrm{A} .8)-(\mathrm{A} .8) G-(\mathrm{A} .8 G)^{\mathrm{T}}$ yields (8.9) and (8.10). Similarly, $\Gamma^{\mathrm{T}} G(\mathrm{~A} .10) \Gamma-(\mathrm{A} .10) \Gamma-(\mathrm{A} .10 \Gamma)^{\mathrm{T}}$ yields $(8.11)$.

Finally, the proof can be reversed so that (8.5)-(8.12) yield (A.1)-(A.6) and (7.2). See [9] for details.

\section{References}

[1] D.S. Bernstein and D.C. Hyland, The optimal projection equations for reduced-order state estimation, IEEE Trans. Automat. Control 30 (1985) 583-585.

[2] S.P. Bhattacharya, The structure of robust observers, IEEE Trans. Automat. Control 21 (1976) 581-588.

[3] J.W. Brewer, Kronecker products and matrix calculus in system theory, IEEE Trans. Circuits and Systems 25 (1978) $772-781$.

[4] S.S.L. Chang and T.K.C. Peng, Adaptive guaranteed cost control of systems with uncertain parameters, IEEE Trans. Automat. Control 17 (1972) 474-483.

[5] R.C. Chung and P.R. Belanger, Minimum-sensitivity filter for linear time-invariant stochastic systems with uncertain parameters, IEEE Trans. Automat. Control 21 (1976) 98-100.

[6] J.A. D'Appolito and C.E. Hutchinson, Low sensitivity filters for state estimation in the presence of large parameter uncertainties, IEEE Trans. Automat. Control 14 (1969) 310-312.

[7] T.E. Djaferis, Robust observers for systems with parameters, Systems Control Lett. 7 (1986) 385-594.

[8] K. Furuta, S. Hara and S. Mori, A class of systems with the same observer, IEEE Trans. Automat. Control 21 (1976) $572-576$.

[9] W.M. Haddad, Robust Optimal Projection Control-System Synthesis, Ph.D. Dissertation, Department of Mechanical Engineering, Florida Institute of Technology, Melbourne, FL (March 1987).

[10] D. Hinrichsen and A.J. Pritchard, Stability radius for structured perturbations and the algebraic Riccati equation, Systems Control Lett. 8 (1987) 105-113.

[11] B.N. Jain, Guaranteed error estimation in uncertain systems, IEEE Trans. Automat. Control 20 (1975) $230-232$.

[12] I.R. Petersen and C.V. Hollot, A Riccati equation approach to the stabilization of uncertain systems, Automatica 22 (1986) 397-411.

[13] I.R. Petersen, A stabilization algorithm for a class of uncertain linear systems, Systems Control Lett. 8 (1987) $351-357$.

[14] R.T. Stefani, Reducing the sensitivity to parameter variations of a minimum-order reduced-order observer, Internat. J. Control 35 (1982) 983-995.

[15] M. Toda and R.V. Patel, Bounds on estimation errors of discrete-time filters under modeling uncertainty, IEEE Trans. Automat. Control 25 (1980) 1115-1121.

[16] A. Vinkler and L.J. Wood, Multistep guaranteed cost control of linear systems with uncertain parameters, J. Guidance Control 2 (1979) 449-456

[17] W.M. Wonham, Linear Multivariable Control: A Geometric Approach (Springer, New York, 1979) 